\title{
Change Detection in SAR Images Based on the Salient Map Guidance and an Accelerated Genetic Algorithm
}

\author{
Caihong $\mathrm{Mu}^{1,2}$, Chengzhou $\mathrm{Li}^{1}$, Yi Liu ${ }^{1}$, Menghua Sun ${ }^{1}$, Licheng Jiao ${ }^{1}$ and $\mathrm{Rong}_{\mathrm{Qu}}{ }^{2}$
}

\author{
${ }^{1}$ Key Laboratory of Intelligent Perception and Image Understanding of Ministry of Education, \\ International Research Center of Intelligent Perception and Computation, International Collaboration Joint Lab in \\ Intelligent Perception and Computation, Xidian University, \\ Xi'an, Shaanxi Province 710071, China \\ ${ }^{2}$ ASAP Research Group, School of Computer Science, \\ University of Nottingham, \\ Nottingham, UK \\ Email: mucaihongxd@ foxmail.com, Caihong.Mu@nottingham.ac.uk
}

\begin{abstract}
This paper proposes a change detection algorithm in synthetic aperture radar (SAR) images based on the salient image guidance and an accelerated genetic algorithm (S-aGA). The difference image is first generated by logarithm ratio operator based on the bi-temporal SAR images acquired in the same region. Then a saliency detection model is applied in the difference image to extract the salient regions containing the changed class pixels. The salient regions are further divided by fuzzy c-means (FCM) clustering algorithm into three categories: changed class (set of pixels with high gray values), unchanged class (set of pixels with low gray values) and undetermined class (set of pixels with middle gray value, which are difficult to classify). Finally, the proposed accelerated GA is applied to explore the reduced search space formed by the undetermined-class pixels according to an objective function considering neighborhood information. In S-aGA, an efficient mutation operator is designed by using the neighborhood information of undetermined-class pixels as the heuristic information to determine the mutation probability of each undeterminedclass pixel adaptively, which accelerates the convergence of the GA significantly. The experimental results on two data sets demonstrate the efficiency of the proposed S-aGA. On the whole, the detection accuracy of S-aGA outperforms five other existing methods including standard GA. In addition, SaGA could obtain satisfying solution within limited generations, converging much faster than standard GA.
\end{abstract}

Keywords-change detection; Saliency map; fuzzy c-means (FCM); genetic algorithm (GA); Synthetic Aperture Radar (SAR) image;

\section{INTRODUCTION}

Synthetic aperture radar (SAR) is able to work through a long distance in all weather with strong penetrating power, so it can get the high resolution information of objects in the harsh environment. At the same time, the technology of SAR image acquisition is becoming more and more advanced, and the SAR image data is increasing gradually, so the SAR images have become an indispensable way of ground observation. Change detection in SAR images is one of the most important methods for SAR image interpretation. It uses two or more SAR images acquired at different time points in the same region to detect the change of the ground. Therefore, change detection in SAR images is widely used in the fields including military reconnaissance, natural disaster assessment, and monitoring on marine oil spill, forest fire and urban development.

Generally, there are two steps for change detection in SAR images: Firstly, the difference image is generated according to the two SAR images acquired at different time points in the same region, and then, both the gray value of the difference image and the relevant neighborhood information are analyzed to get the change map. The traditional methods of difference image analysis include threshold segmentation and clustering [1]. Threshold segmentation method [2] is simple and fast, but its classification accuracy is not high. K-means is one of the classical clustering methods, which updates suitable clustering centers iteratively, and then divides the pixels into changed and unchanged classes based on the idea of hard partition. Fuzzy c-means (FCM) clustering introduces the idea of fuzzy set to the determination of the cluster center, and generates the membership matrix, which has become a popular clustering method.

The presence of multiplicative speckle noise in SAR images increases the difficulty of SAR-image change detection. The detection accuracy could be improved by the consideration of neighborhood information in the analysis of the difference image. Krinidis et al. [3] proposed the fuzzy local information C-Means (FLICM). By adding fuzzy local spatial information and gray information, the problem of noise sensitivity and detail retention can be balanced, and the method does not need to set any parameters artificially. Gong et al. [4] proposed an improved clustering method by adding the MRF method to the process of FCM, which is named as MRFFCM. The MRFFCM improved FCM by modifying the membership of each pixel according to the MRF-based spatial. The spatial context is contained in the pivotal energy function through the use of the neighborhood system. The form of the energy function is altered by utilizing not only the memberships but also the number of the same class of 
neighborhood pixels.

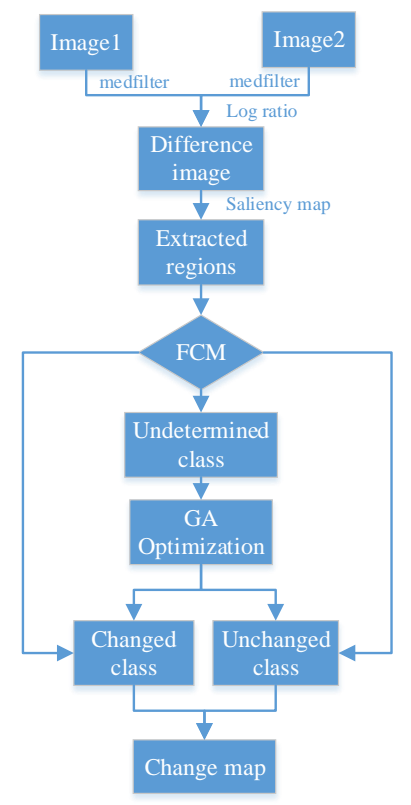

Fig.1 Flowchart of S-aGA

Most of the above methods analyze the difference image by searching for the appropriate clustering centers so as to obtain a change map. In fact, the analysis of the difference image is a binary classification problem, in which the pixels in the difference image are marked with the changed class label and the unchanged class label. Assume the changed class is labeled as 1, and unchanged class is labeled as 0 . The change map is simply a matrix of binary numbers [1]. Celik [5] pointed out that the genetic algorithm (GA) can be employed to find the final change detection mask by evolving the initial binary change detection mask through generations. The proposed method is able to produce the change detection result in the process of modeling the difference image data without a parameter tuning or priori assumptions. Therefore, this method is a general-purpose unsupervised change detection method. GA $[5,6]$ is characterized by high efficiency, parallelism and global search, which can automatically acquire and accumulate knowledge about the search space in the search process, and control the search process adaptively to obtain the optimal solution. However, when using GA to analyze the difference image and determine the label for each pixel is 1 or 0 , for a difference image with the size of $500 \times 500$, the size of the search space of the GA will be $2^{250000}$. The search space will grow exponentially with the increasing of the size of difference image, which leads to that the evolution of GA becomes too slow to obtain the satisfying solution. This has become the bottleneck of GA when it is applied in the problem of image processing. In addition, the method proposed by Celik [5] only uses the gray value of the difference image, without considering the neighborhood information, so it may fail to get a satisfactory detection result for the SAR image with strong noise.

As mentioned above, the GA can be well adapted to analyze the difference image to obtain a binary matrix change map. However, two important issues must be addressed: how to reduce the computational cost, and how to solve the noise-sensitive problem.

For the first problem, that is, how to reduce the computational cost, we consider to deal with it by reducing the search space. The saliency detection model could be used here. At any given time, not all areas and content are captured by the human visual system, and the human vision system will automatically select the regions of interest in all regions [7]. A saliency detection model is proposed to extract the salient regions of the image using the characteristics of the human visual system. Therefore, we can utilize this visual attention mechanism to determine the rough location of the changed area, thus greatly reducing the search space. In addition, during the classification of the difference image, the class labels of pixels with middle gray values are not easy to determine. FCM is used to classify the pixels of salient regions into three categories: changed class, unchanged class and undetermined class. Then the proposed accelerated GA is applied to explore the reduced search space formed by the undetermined-class pixels. In addition, in the proposed accelerated GA, the prior knowledge of the neighborhood correlation of difference image can be used to provide heuristic information to determine the mutation probability of each undetermined-class pixel adaptively, which accelerates the convergence of the GA significantly and further reduce computational cost of GA.

For the second problem, i.e., how to solve the sensitive problem, we consider the neighborhood information in the objective function of the GA for balancing noise suppression and detail preserving. In addition, the use of the prior knowledge of the neighborhood correlation of difference image in the mutation operation of GA could also improve the ability of reducing noise.

Based on the above ideas, this paper proposed a SARimage change detection algorithm based on the salient map guidance and an accelerated GA (S-aGA). The main contributions of this paper are as follows: (1) The search space are reduced by effective methods, which greatly reduces the computational cost of GA. (2) The constraint of neighborhood information is added in the objective function, which is used to balance the noise suppression and detail reservation. (3) The neighborhood information in difference image is used as the heuristic information for the mutation operator design, thus accelerating the convergence rate of the algorithm and improving the antinoise ability of the detection algorithm. The abovementioned measures can effectively alleviate the bottleneck problem of GA applied to SAR-image change detection. Fig. 1 shows the flowchart of the algorithm proposed in this paper. The flowchart has three main parts. First, the salient regions are extracted by the saliency detection model. Then, FCM is used to classify the pixels of the salient regions into the changed class, the unchanged class and the undetermined class. Finally, the accelerated GA is used to classify the pixels in the undetermined class.

The remainder of the paper will be organized as follows: Section 2 presents the details of the proposed method. Section 3 describes the experimental results of the proposed algorithm and compares it with the existing methods. Section 4 summarizes the paper.

\section{PROPOSED CHANGE DETECTION METHOD}

Consider two SAR images acquired at different times in the same region that have been registered:

$$
I_{1}=\left\{I_{1}(i, j) \mid 1 \leq i \leq H, 1 \leq j \leq W\right\}
$$




$$
I_{2}=\left\{I_{2}(i, j) \mid 1 \leq i \leq H, 1 \leq j \leq W\right\}
$$

Where the size of the SAR image is $H \times W$. Considering the presence of multiplicative speckle noise in SAR images, the images are first processed by median filter, and the difference image $D I$ is generated by logarithm ratio operator:

$$
D I=\left|\log \frac{I_{1}+1}{I_{2}+1}\right|=\left|\log \left(I_{1}+1\right)-\log \left(I_{2}+1\right)\right|
$$

Where $\log$ is the natural logarithm. Then, after the difference image is analyzed, the final binary matrix change map $C M=\{C M(\mathrm{i}, \mathrm{j}) \mid 1 \leq i \leq H, 1 \leq j \leq W\}$ is obtained, in which label 1 represents the corresponding pixel belongs to the changed class, and label 0 represents the corresponding pixel belongs to the unchanged class. The following describes how to analyze the difference image to get the change map.

\section{A. Salient Regions Extraction}

As the introduction mentioned, saliency detection is inspired by the human visual system. The human eyes reduce the computational complexity and obtain useful information by automatically capturing regions of interest. Using this feature, a method was proposed based on machine vision for image saliency detection [8], which uses the contrast of gray values between the highlighted object and the background, and compares the local and global neighborhoods to capture salient regions. Saliency detection is to find significant areas in the image, and SARimage change detection is to analyze the difference image to find out the great changes in the pixel. Therefore, we use a saliency detection model to extract the region of interest in the difference image, thereby reducing the search space of GA.

In this paper, we use context-ware saliency detection method proposed in [8]. The image is divided into patches, and then the similarities between each patch and other patches in the entire image are measured to obtain the local and global saliency of the given patch. The multi-scale method is used to calculate the saliency of each patch, and finally, salient regions are obtained which are much different from other regions. We adopt the parameter setting of the proposed method in [8]. For more details of saliency extraction, one can refer to the paper [8].

After obtaining the saliency map of the difference map, we use the maximum interclass variance method OTSU for automatic segmentation to obtain the binarized saliency map $S \in R^{H \times W}$, which is defined in (4):

$$
\begin{aligned}
& S(i, j)= \begin{cases}1, & \text { salient regions } \\
0, & \text { non-salient regions }\end{cases} \\
& 1 \leq i \leq H, 1 \leq j \leq W
\end{aligned}
$$

Therefore, the difference image DIS is obtained which only contains the salient regions, that is,

$$
D I S=D I\{(i, j) \mid S(i, j)=1,1 \leq i \leq H, 1 \leq j \leq W\}
$$

\section{B. FCM Pre-classification}

After the salient regions containing the changed classes is obtained, pixels whose class need to be determined in the difference image could be reduced to a great extent, which leads to reduction of the search space for the GA. In the salient regions, there are three types of pixels: high-grayscale changed class pixels, low-gray-scale unchanged class pixels and undetermined class pixels in the middle gray level. The correct classification of undetermined class pixels has a great effect on the results of change detection method.

Based on this idea, we first use the traditional FCM algorithm to divide the pixels of the salient regions DIS into three classes: changed class $\omega_{c}$, unchanged class $\omega_{u c}$ and undetermined class $\omega_{u d}$. The search space is reduced further by restricting the search space of GA within the set of the pixels of undetermined class. The following is a detailed description of the FCM-based pre-classification method proposed in this paper.

- Input: all pixels of the salient regions DIS.

- Generate the membership matrix: The FCM algorithm divides all the pixels in the DIS into three classes. The objective function is set as follows:

$$
\begin{aligned}
& \min \quad J_{m}=\sum_{i=1}^{c} \sum_{j=1}^{N} u_{i j}^{m}\left\|x_{j}-v_{i}\right\|^{2} \\
& \text { s.t. } \quad \sum_{i=1}^{c} u_{i j}=1 \quad u_{i j} \in[0,1], \forall j
\end{aligned}
$$

Where $D I S=\left\{x_{1}, x_{2}, \cdots, \mathrm{x}_{N}\right\}$ is the set of all the pixels in the salient regions, and $N$ is the number of pixels in the salient regions. $c$ denotes the number of cluster centers, and here, $c=3 . U=\left[u_{i j}\right], U \in \mathrm{R}^{c \times N}$, which is the membership degree matrix, representing the membership of a pixel belonging to the $i^{\text {th }}$ cluster. $m$ denotes the weighting index of each fuzzy member. $v_{i}$ denotes the gray value of the $i^{\text {th }}$ cluster center. By iteration, the membership degree $u_{i j}$ and clustering center $v_{i}$ are updated till the algorithm converges.

1) Set the parameters $c=3, m=2$, threshold $\varepsilon$, initial the membership matrix $U^{0}$, and then set counter $t=1$.

2) The gray value of $c$ cluster centers $v_{i}^{t}$ is calculated using (7).

$$
v_{i}^{t}=\frac{\sum_{j=1}^{N}\left(u_{i j}^{t}\right)^{m} x_{j}}{\sum_{j=1}^{N}\left(u_{i j}^{t}\right)^{m}}, i=1,2,3 .
$$

3) The membership matrix $U^{t}$ is calculated as (8).

$$
u_{i j}^{t}=\frac{1}{\sum_{k=1}^{c}\left(\frac{\left\|x_{j}-v_{i}^{t}\right\|^{2}}{\left\|x_{j}-v_{k}^{t}\right\|^{2}}\right)^{1 /(m-1)}}
$$

4) If $\max \left\{U^{t}-U^{t-1}\right\}<\varepsilon$, the algorithm stops. Otherwise $t=t+1$, and then go to step 2).

According to the membership matrix, each pixel is classified into one of the class set $\left\{\omega_{c}, \omega_{u c}, \omega_{u d}\right\}$, and set 


$$
\begin{aligned}
& \text { num1 }=\arg _{k}\left\{\max \left\{v_{k}\right\}\right\}, \\
& \text { num2 }=\arg _{k}\left\{\min \left\{v_{k}\right\}\right\}, k=1,2,3 .
\end{aligned}
$$

Then,

$$
C_{j}=\left\{\begin{array}{l}
\omega_{c}, \quad \text { if } \text { num } 1=\arg _{k}\left\{\max \left\{u_{k j}\right\}\right\}, \max \left\{u_{k j}\right\}>\tau \\
\omega_{u c}, \quad \text { if num } 2=\arg _{k}\left\{\max \left\{u_{k j}\right\}\right\}, \max \left\{u_{k j}\right\}>\tau(9) \\
\omega_{u d}, \quad \text { otherwise }
\end{array}\right.
$$

Here, $\tau$ is the threshold value of the membership degree, and $\tau=0.90$ which is set to ensure that the pixels marked as the changed class or the unchanged class have high membership degree with the corresponding clustering center.

\section{Accelerated GA}

By the previous process, the problem of obtaining the change map from the difference image can be transformed into the problem of using the GA to assign class labels for the undetermined class pixels. In this way, the narrowing of the GA search space is realized, which makes it possible to detect the changes of SAR image rapidly.

However, there is another problem. If the size of the difference image is large, and the number of pixels contained in the undetermined class within the salient regions is also large, the search space of the GA will still be large. In this paper, we propose an accelerated GA based on neighborhood information which is used as the heuristic information for algorithm design in SAR-image change detection. This algorithm is characterized by the strong ability of anti-noise and fast convergence. The proposed accelerated GA has three improvements compared with the standard GA: First of all, in order to utilize the spatial neighborhood of the pixels of the change map, the individual is encoded in two-dimensional coding which can effectively preserve the individual spatial structure information. Then, the neighborhood information is introduced into the objective function to improve the antinoise effect of GA change detection to some extent. Finally, the neighborhood information of undetermined class pixels in the salient regions is used as the heuristic information to guide the determining of the mutation probability of the undetermined class of pixels, thus accelerating the convergence of the GA. The following will introduce these three aspects in detail.

- Binary two-dimensional encoding method

In order to preserve the spatial structure of the individuals, the binary two-dimensional matrix encoding method is used to construct the individual, and the size of the matrix is $\mathrm{H} \times \mathrm{W}$ which is the same with that of the difference image. During the initialization of each individual, the points of the non-salient regions detected by the saliency detection model and the unchanged class pixels determined by the FCM in the salient regions are encoded as 0 , and no further genetic manipulation is performed at these points. The points of the changed class determined by the FCM in the salient regions are encoded as 1 , and no genetic manipulation is performed at these points either. Finally, 0 or 1 labels are randomly assigned to the points of the undetermined classes determined by the FCM in the salient regions, and the genetic manipulation will be executed at these points to search for the optimal solution.

\section{- Objective functions with neighborhood information}

To find the optimal solution, an objective function similar to (6) should be proposed, in which the neighborhood information should be considered. Let $C M$ represent an individual. The objective function of $C M$ is $O F$, then the fitness value Fitness $=\frac{1}{O F} . O F$ is calculated according to (10) and (11):

$$
\begin{aligned}
& O F=\frac{1}{H \times W} \sum_{r=0}^{1} M_{r} \sum_{\forall(i, j) \in \mathrm{R}_{\mathrm{r}}}\left[\left(D I S(i, j)-v_{\mathrm{r}}\right)^{2}+G_{r}(i, j)\right] \\
& G_{r}(i, j)=\frac{1}{Z} \sum_{\substack{k \in N_{i j} \\
k \neq(i, j)}} \frac{1}{d_{k}+1} \times\left(1-u_{k r}\right)^{2} \times\left(D I S_{k}(i, j)-v_{\mathrm{r}}\right)^{2}
\end{aligned}
$$

Where $G_{r}(i, j)$ is the neighborhood constraint term at the point $(i, j)$. Here, $r \in\{0,1\}, R_{0}$ represents all unchanged class pixels, that is: $R_{0}=\{(i, j) \mid \mathrm{CM}(i, j)=0\}$. $R_{1}$ represents all the pixels of the changed class, that is: $R_{1}=\{(i, j) \mid \mathrm{CM}(i, j)=1\} \quad . \quad N_{i j} \quad$ denotes a $3 \times 3$ neighborhood of the point $(i, j)$ in $D I S$, and $D I S_{k}(i, j)$ represents the $k^{\text {th }}$ point of the $N_{i j}$ of point $(i, j) . d_{k}$ denotes the spatial distance between the $k^{\text {th }}$ point in the neighborhood and the center point $(i, j) . M_{r}$, the count statistic of pixels of changed class and unchanged class, $v_{r}$, the gray value of the $r^{\text {th }}$ cluster center, and the normalization weight of spatial distance $Z$ are defined as follows:

$$
\begin{gathered}
M_{r}=\sum_{\forall(i, j) \in R_{r}} 1, \quad r \in\{0,1\} \\
v_{r}=\frac{1}{M_{r}} \sum_{\forall(i, j) \in R_{r}} D I S(i, j), \quad r \in\{0,1\} \\
Z=\sum_{\substack{k \in N_{i j} \\
k \neq(i, j)}} \frac{1}{d_{k}+1}
\end{gathered}
$$

The first term of the objective function is the intra class variance of $D I S$. The second term $G_{r}(i, j)$ is the neighborhood information of the point $(i, j)$, which is inspired by the neighborhood information formula of the objective function in the FLICM method [3]. $\frac{1}{d_{k}+1}$ represents the influence weight determined by the spatial distance between the neighborhood pixel and the center pixel, and the larger the distance, the smaller the weight. $\frac{1}{Z}$ is the normalization of the weight. $u_{k r}$ is the degree of membership of the $k^{\text {th }}$ pixel in the $r^{\text {th }}$ cluster, and $u_{k r}$ is defined as (15).

$$
u_{k r}=1-\frac{\left(\left(D I S_{k}(i, j)-v_{\mathrm{r}}\right)^{2}\right.}{\left(\left(D I S_{k}(i, j)-v_{1}\right)^{2}+\left(\left(D I S_{k}(i, j)-v_{2}\right)^{2}\right.\right.}
$$

- Computation of mutation probability based on neighborhood information 
In standard GA, the probability of mutation remains unchanged, and the genes on which the mutation is executed are randomly determined, which is named as traditional mutation operator here. However, during the process of obtaining the change map from the difference image, it is blind by using the traditional mutation operator to change the class label of the undetermined class pixels. When the number of the undetermined class pixels is very large, using traditional mutation operator will make the algorithm converge slow.

Considering the association between the neighborhood and the central pixel in $D I S$, we propose a novel mutation operator using the neighborhood information of DIS as the heuristic information. For each pixel of the undetermined class in the individual, if the classification tag at the $(x, y)$ of $C M$ is denoted as $C M(x, y)$, according to the neighborhood information of the pixels of DIS in the position of $(x, y)$, we can compute the mutation probability $P_{m}(x, y)$ of $C M(x, y)$ adaptively. The following are the steps for computing the mutation probability.

Step 1: The $3 \times 3$ neighborhood of $(x, y)$ in DIS is denoted as $L_{(x, y)}$. For $L_{j} \in L_{(x, y)}, j=1,2,3, \ldots, 9$, the membership degree $u_{j 0}$ and $u_{j 1}$ of the neighboring pixels belonging to individual cluster centers $v_{0}$ and $v_{1}\left(v_{0}\right.$ and $v_{1}$ are obtained according to (13)) are calculated using (16), respectively.

$$
u_{j r}=\frac{1}{\sum_{r=0}^{1} \frac{\left(L_{j}-v_{r}\right)^{2}}{\left(L_{j}-v_{r}\right)^{2}}}, r=0,1
$$

Step2: Through the membership of the neighborhood $L_{(x, y)}$, class $C_{j}$ of each neighborhood pixel is determined as (17).

$$
C_{j}= \begin{cases}1 & u_{j 1}>u_{j 0} \\ 0 & u_{j 0}>u_{j 1}\end{cases}
$$

Step3: Calculate the mutation probability $P_{m}(x, y)$ of $C M(x, y)$,

$$
\begin{gathered}
P_{m}(x, y)=p \times Z^{2} \\
Z=C M(x, y) \times \sum_{\substack{j \in L_{(x, y)} \\
j \neq(x, y)}} \frac{1}{d_{j}}-\sum_{\substack{j \in L_{(x, y)} \\
j \neq(x, y)}} \frac{1}{d_{j}} C_{j}
\end{gathered}
$$

Where $\mathrm{p}$ is the basic mutation probability, and $p=0.0001 \sim 0.001 . d_{j}$ is the spatial distance between the neighborhood element and the center point. The distance between the element in the four neighborhood and the center point is 1 , and the distance between the diagonal element and the center point is $\sqrt{2}$. By using the neighborhood information of the difference image, the parameter $Z$ can be determined, and $|Z| \in[0,4+2 \sqrt{2}]$. The mutation probability $P_{m}(\mathrm{x}, \mathrm{y})$ of the undetermined class pixels in the individual can be changed adaptively according to the class of the neighborhood pixel $L_{(x, y)}$. When $C M(x, y)$ is consistent with the class of majority of the neighborhood $L_{(x, y)}$, the mutation probability becomes smaller to maintain the original region consistency. Otherwise, the mutation probability increases, so that $C M(x, y)$ tends to become consistent with the majority of the neighborhood $L_{(x, y)}$. This can speed up the GA to search for the optimal solution process significantly.

The process of accelerated GA in this paper is as follows:

1) Generation of initial population. The population consists of $K$ individuals, each of which is binary encoded in two dimensions. The size of the individual is $H \times W$ (consistent with the size of the difference image). Set the evolutionary number counter $g=1$.

2) The fitness values of the individuals are calculated according to (10).

3) The offspring are selected by tournament selection method, with the elite retention strategy.

4) Uniform crossover is applied to the offspring.

5) The mutation probability $P_{m}(\mathrm{x}, \mathrm{y})$ of the undetermined class pixels in the individual is computed, according to (18) and (19), and then the mutation operation is performed.

6) Set $g=g+1$. If the termination condition is satisfied, then the algorithm is terminated, and the elite individual of current generation is output as the final change map result. Otherwise, go to step 2).

\section{EXPERIMENTS}

In this section, to evaluate the performance of the proposed method S-aGA, five algorithms are implemented for comparison. They are FCM, FLICM [3], MRFFCM [4], a method of combining the saliency map with the FCM (SFCM), and standard GA [5] (denoted as GA).

The false negatives ( $F N$, changed pixels that undetected), the false positives ( $F P$, unchanged pixels are wrongly classified as changed), overall error $(O E$, the total number of $F N$ and $F P$ ), the percentage correct classification (PCC), and Kappa, will be used as the evaluation criteria

All experiments were performed on a personal computer and the configuration parameters are as follows: Core(TM)i7-4720HQ CPU, 8GB RAM. Matlab is the software employed in these experiments, and the Matlab function tic and toc are used to estimate the detection time.

\section{A. Introduction of Experimental Data Set}

The first data set is the Bern dataset, which contains two registered images obtained in the city of Bern, Switzerland, acquired by the European Remote Sensing 2 satellite SAR sensor April and May 1999, respectively. The size of the image is $301 \times 301$. The data are SAR images of the area before and after floods. In May 1999, River Aare flooded parts of the cities of Thun and Bern and the airport of Bern entirely. The images captured at these two time points can be used to detect areas where flooding occurred. The images of this dataset and the available reference image are shown in Fig. 2 .

The second dataset is the Ottawa dataset, which contains two registered SAR images with an image size of $290 \times 350$, acquired by the RADARSAT SAR sensor in the city of Ottawa, in May and August 1997, respectively. They 
are provided by the Defence Research and Development Canada, Ottawa. The two images reflected the impact of flooding in this area. The image of this dataset and the reference image are shown in Fig.3.

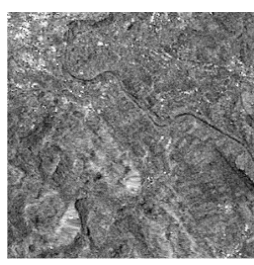

(a)

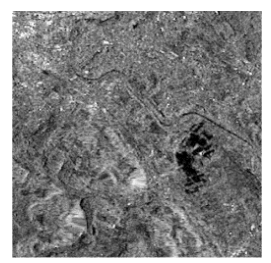

(b)

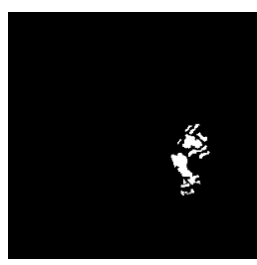

(c)
Fig.2 The Bern dataset used in the experiments. (a) SAR image acquired in April 1999. (b) SAR image acquired in May 1999 after flood. (c) The reference image.

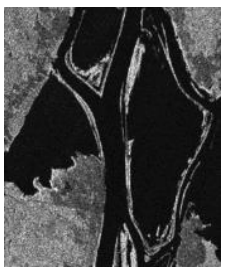

(a)

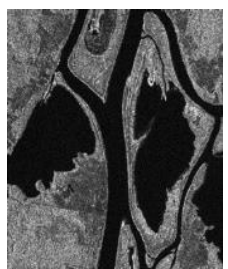

(b)

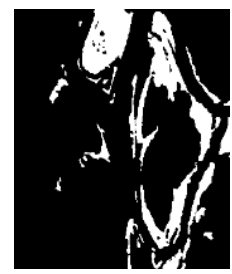

(c)
Fig. 3 The Ottawa dataset used in the experiments. (a) SAR image acquired in May 1997. (b) SAR image acquired in August 1997. (c) (c) The reference image.

\section{$B$ experimental results}

In the experiment, the parameters of the proposed method S-aGA are set as follows: the number of individuals $K=20$, the uniform crossover probability $P_{c}=0.8$, and the base mutation probability $p=0.0001$ in mutation operation. For standard GA, which is implemented according to the description of [5], the parameters are set the same with those of [5], i.e., the number of individuals $K=20$, crossover probability $P_{c}=0.8$, and the mutation probability $P_{m}=0.01$. The objective function of standard GA is also the same with that of [5].

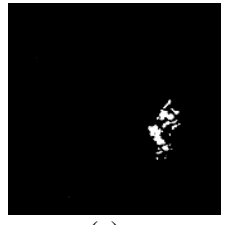

(a)

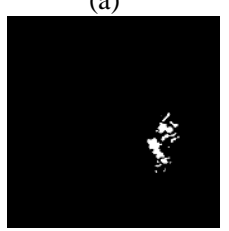

(d)

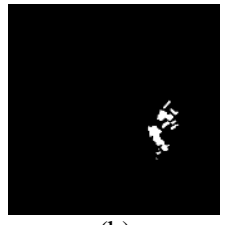

(b)

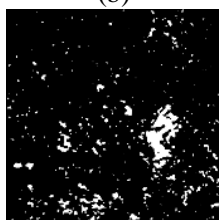

(e)

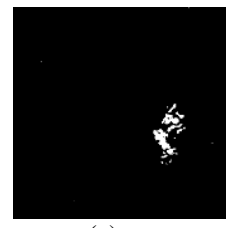

(c)

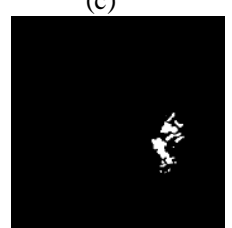

(f)
Fig.4. Change detection results by using different methods on the Bern dataset. (a) FCM, (b) FLICM, (c) MRFFCM, (d) S-FCM, (e) GA by iterating $2 \times 10^{5}$ times, (f) S-aGA.

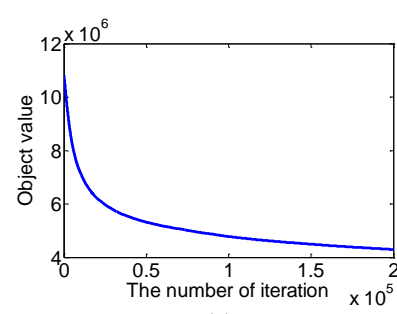

(a)

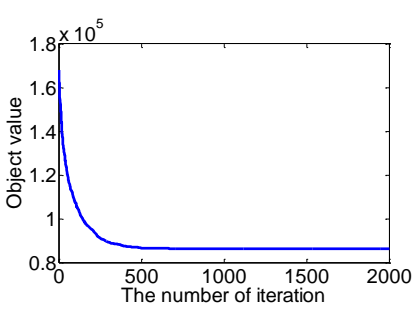

(b)
Fig.5. The convergence curve on the Bern dataset. (a) GA by iterating $2 \times 10^{5}$ times .(b) S-aGA.

TABLE I. VALUES OF THE EVALUATION CRITERIA OF THE BERN DATASET

\begin{tabular}{|l|l|l|l|l|l|}
\hline \multicolumn{1}{|c|}{ Criteria } & $\boldsymbol{F N}$ & $\boldsymbol{F P}$ & $\boldsymbol{O E}$ & $\boldsymbol{P C C}$ & Kappa \\
\hline FCM & 315 & 42 & 357 & 0.9961 & 0.8228 \\
\hline FLICM & 293 & 36 & 329 & 0.9964 & 0.8379 \\
\hline MRFFCM & 63 & 281 & 344 & 0.9962 & 0.8337 \\
\hline S-FCM & 306 & $\mathbf{4 1}$ & 347 & 0.9962 & 0.8284 \\
\hline GA & $\mathbf{2 4}$ & 4513 & 4537 & 0.9499 & 0.3183 \\
\hline S-aGA & 188 & 91 & $\mathbf{2 7 9}$ & $\mathbf{0 . 9 9 6 9}$ & $\mathbf{0 . 8 7 2 4}$ \\
\hline
\end{tabular}

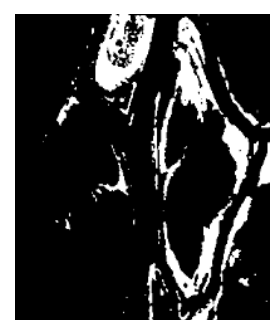

(a)

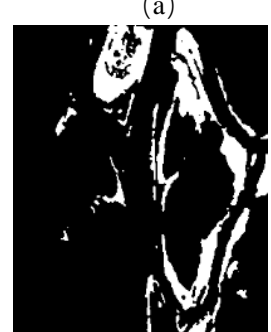

(d)

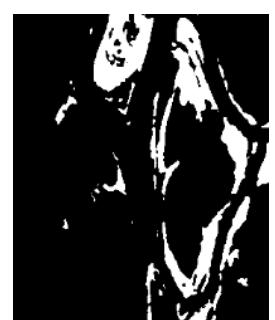

(b)

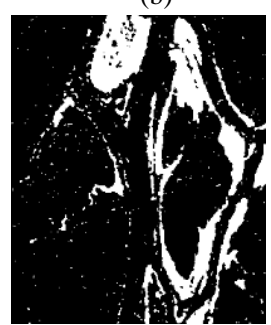

(e)

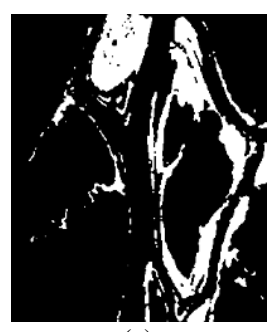

(c)

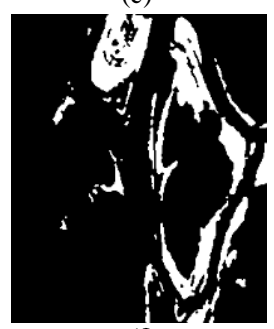

(f)
Fig.6. Change detection results by using different methods on the Ottawa dataset. (a) FCM, (b) FLICM, (c) MRFFCM, (d) S-FCM, (e) GA by iterating $2 \times 10^{5}$ times, (f) S-aGA.

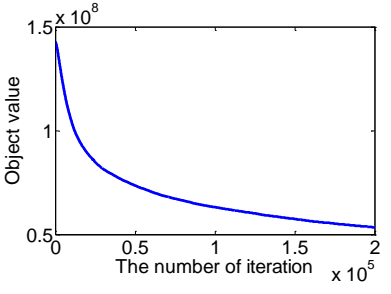

(a)

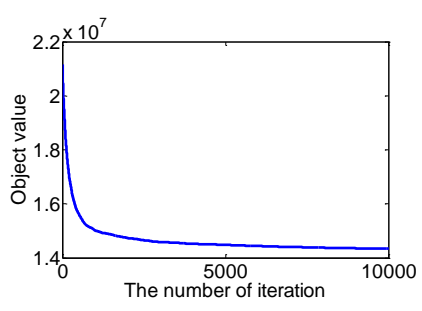

(b)
Fig.7. The convergence curve on the Ottawa dataset. (a) GA by iterating $2 \times 10^{5}$ times .(b) S-aGA.

TABLE II. VALUES OF THE EVALUATION CRITERIA OF THE OTTAWA DATASET

\begin{tabular}{|l|l|l|l|l|l|}
\hline Method & $\boldsymbol{F N}$ & $\boldsymbol{F P}$ & $\boldsymbol{O E}$ & $\boldsymbol{P C C}$ & Kappa \\
\hline FCM & 2455 & 378 & 2833 & 0.9721 & 0.8893 \\
\hline FLICM & 2080 & $\mathbf{1 7 0}$ & 2250 & 0.9778 & 0.9125 \\
\hline MRFFCM & 1703 & 666 & 2369 & 0.9769 & 0.9146 \\
\hline S-FCM & 2360 & 220 & 2580 & 0.9746 & 0.8991 \\
\hline GA & $\mathbf{8 1 1}$ & 2818 & 3629 & 0.9642 & 0.8722 \\
\hline S-GA & 1712 & 382 & $\mathbf{2 0 9 4}$ & $\mathbf{0 . 9 7 9 4}$ & $\mathbf{0 . 9 1 9 8}$ \\
\hline
\end{tabular}

Fig. 4 and Table I. show the experimental results of the Bern dataset, by using six algorithms respectively. The proposed method S-aGA achieves the minimum overall error $(O E=279)$, and the maximum Kappa coefficient $($ Kappa $=0.8724)$, which is superior to other methods in this data set. In FCM method and S-FCM method, only the gray values of the pixels are used as clustering information, so that they are much weaker on noise suppression than other methods which use local neighborhood information. Therefore, the false negatives $F N$ of these two methods are higher. This shows that the 
use of local neighborhood information can help to obtain more accurate changes. In addition, the S-FCM method uses a saliency detection model to obtain salient regions which contain changed regions, so that the pixels of nonsalient regions cannot interfere the detection of changes. Hence, the detection accuracy is improved. The S-aGA takes the local neighborhood information into consideration in both the objective function and the mutation probability, and has a better effect on noise suppression, the performance of which is greatly improved compared with the standard GA.

Fig. 5 demonstrates the convergence curves of GA and S-aGA on the Bern dataset, respectively. Fig.5. (b) shows S-aGA iterates $2 \times 10^{3}$ times to reach convergence. Fig.5. (a) shows the standard GA method did not converge after $2 \times 10^{5}$ iterations. Therefore, S-aGA method reduces the essential iterations by more than 100 times. In terms of running time, $\mathrm{S}$-aGA uses 12 minutes, reducing more than 122 times compared with standard GA using 1466 minutes. Obviously, the S-aGA method accelerates the convergence rate of change detection.

Fig. 6 and Table II. show the results of six algorithms tested on the Ottawa dataset, respectively. In this data set, S-aGA obtained the best result in terms of $O E, P C C$ and Kappa coefficient, i.e., $O E=2094, \quad P C C=0.9794$, Kappa $=0.9198$. Compared with other methods, the detection results of the proposed method have improved to some extent. The methods using local neighborhood information, i.e., FLICM, MRFFCM, and S-aGA are better than FCM, S-FCM and GA which only use gray values of pixels. In the S-FCM method, the saliency detection model is used to reduce the noise interference in the change detection to a certain extent, and the detection result is better than the FCM method.

Fig. 7 shows the convergence curves of the standard GA and S-aGA on the Ottawa dataset, respectively. S-aGA could obtain good results with $1 \times 10^{4}$ evolutions. Fig7. (b) is the result of standard GA method with $2 \times 10^{5}$ evolution times, but its objective function is still very high. Compared with the standard GA, S-aGA method reduces the iterations by more than 20 times. In the run-time, the standard GA takes about 1652 minutes, and the S-aGA uses 85 minutes which is about 20 times lower. It is proved that the proposed S-aGA method is effective.

It could be found the proposed S-aGA performs well on change detection, which realizes the purpose of rapid detection based on genetic algorithm. In the above two data sets, the image size of the Bern dataset is $301 \times 301$, the number of pixels in the salient regions is 3628 , and finally the number of the undetermined class pixels classified by FCM is 1244 . The image size of the Ottawa dataset is 290 $\times 350$, the total number of pixels in the salient regions is 40525 , and the number of the undetermined class pixels classified by FCM is 14119 . With the use of saliency detection model and FCM pre-classification, the proposed method effectively reduces the search space. However, due to the increase of the salient area in the Ottawa dataset, the number of undetermined class pixels is about 11.3 times compare with Bern dataset, and the search space is increased by $2^{12875}$ times. Here, the acceleration strategy proposed in the GA plays an important role in speeding up convergence of the algorithm. From the convergence curve in Fig.7 (b) of the S-aGA, it could be observed that when the number of undetermined class pixels increases, the algorithm of this paper can still achieve fast detection.

\section{CONCLUSIONS}

A new method based on salient map guidance and accelerate $\mathrm{GA}(\mathrm{S}-\mathrm{aGA})$ is proposed for SAR-image change detection. Firstly, a saliency map containing the changed regions is extracted by using the saliency detection model based on the feature of human visual attention, and then the search space of GA can be reduced to the salient regions. Next, the FCM algorithm is used for pre-classification. The pixels of the salient regions are divided into three categories: changed class, unchanged class and undetermined class, and the search space of GA is reduced further to the set of the undetermined class pixels. Finally, an accelerated GA method is proposed. In the accelerated GA, a mutation operator is designed by considering the neighborhood information of the difference image, which accelerates the convergence of the GA significantly. In addition, the neighborhood information is added into the objective function to balance the noise suppression and detail preserving problems. The experimental results show that the proposed S-aGA is superior to the standard GA for SAR-image change detection. The experimental results show that S-aGA has the advantages of excellent detection performance and strong noise suppression capability compared with existing methods, as well as a significant improvement in SAR-image detection speed relative to the simple GA-based approach.

In this paper, the proposed S-aGA does not need to set any artificial parameters. The addition of neighborhood information in mutate operator accelerates the convergence speed of the algorithm, which alleviates the problem that the standard GA method has too high computational cost in the pixel-based image classification problem, and makes the algorithm have strong noise suppression ability. Furthermore, The application range of accelerated GA method can be applied not only to the binary classification problem of change detection, but also to multiclassification scenarios of other images, which would be studied in the future.

\section{ACKNOWLEDGMENT}

This work was supported by the National Basic Research Program (973 Program) of China (No. 2013CB329402), the National Natural Science Foundation of China (Nos. 61672405, 61373111, 61573015, 61473215 and 61371201), the Fundamental Research Funds for the Central Universities (No. JBG160229), the Fund for Foreign Scholars in University Research and Teaching Programs (the 111 Project) (No. B07048), the Major Research Plan of the National Natural Science Foundation of China (Nos. 91438201 and 91438103), the Program for Cheung Kong Scholars and Innovative Research Team in University (No. IRT_15R53) and China Scholarship Council (CSC).

\section{REFERENCES}

[1] W. Ren, J. Song, S. Tian, and W. Wu, "Survey on unsupervised change detection techniques in SAR images1," Signal and Information Processing (ChinaSIP), 2014 IEEE China Summit \& International Conference on. IEEE, 2014, pp. 143-147

[2] M. N. Sumaiya and R. Shantha Selva Kumari, "Logarithmic MeanBased Thresholding for SAR Image Change Detection," IEEE Geoscience and Remote Sensing Letters, vol. 13, no. 11, pp. 17261728, November 2016. 
[3] S. Krinidis, and V. Chatzis, "A robust fuzzy local information Cmeans clustering algorithm," IEEE Transactions on Image Processing, vol. 19, no. 5, pp. 1328-1337, May 2010.

[4] M. Gong, L. Su, M. Jia, and W. Chen, "Fuzzy clustering with a modified MRF energy function for change detection in synthetic aperture radar images," IEEE Transactions on Fuzzy Systems, vol. 22, no. 1, pp. 98-109, February 2014.

[5] T. Celik, "Change detection in satellite images using a genetic algorithm approach," IEEE Geoscience and Remote Sensing Letters, vol. 7, no. 2, pp. 386-390, April 2010

[6] C. Mu, J. Xie and Liu Y, et al. "Memetic algorithm with simulated annealing strategy and tightness greedy optimization for community detection in networks," Applied Soft Computing, 2015, vol. 34, pp. 485-501.
[7] D. Liu, J. Zhang, and X. Lu, "An unsupervised automatic change detection approach based on visual attention mechanism," Geoscience and Remote Sensing Sympos ium (IGARSS), 2015 IEEE International. IEEE, 2015, pp. 3045-3048.

[8] S. Goferman, L. Zelnik-Manor, and A. Tal, "Context-aware saliency detection," IEEE Transactions on Pattern Analysis and Machine Intelligence, vol. 34, no. 10, pp. 1915-1926, October 2012. 